\title{
Medication Bezoar Causing acute Gastric Outlet Obstruction: A Case Report
}

\author{
MA MAZID
}

\begin{abstract}
Summary:
Medication bezoars are rare and are composed of medications and/or medication vehicles. Rarely, medication bezoars can cause serious problems due to complications such as perforation, obstruction, haemorrhage. A 60 years old woman presented with 10 days history of epigastric pain, weakness and postprandial non-bilious vomiting. Her abdominal ultrasonography showed strong post acoustic shadow noted within 1st part of duodenum possibly foreign body. Upper gastrointestinal endoscopy was performed and a bezoar of tablet of aluminum hydroxide was extracted. The patient had uneventful recovery.
\end{abstract}

\section{Introduction:}

Medication bezoars are unusual entities. Medications reported to cause bezoars include aluminum hydroxide gel, enteric-coated aspirin, sucralfate, guar gum, cholestyramine, enteral feeding formulas, psyllium preparations, nifedipine XL, and meprobamate ${ }^{1}$. These patients often present with signs and symptoms consistent with an obstruction of the gastrointestinal tract and represent an even greater diagnostic challenge due to the rarity of this complication. To date, treatment of medication bezoars involves mainly physical manipulation of the bezoar through lavage, endoscopic removal, or, in most cases, surgical removal $^{2}$. Herein, a 60 years old lady who presented with acute gastric outlet obstruction and had an uneventful recovery after endoscopic extraction of medication bezoar.

\section{Presentation of the case}

A 60 years old woman presented with 10 days history of epigastric pain, weakness and postprandial nonbilious vomiting. Pain did not radiate, and was not associated with jaundice, fever or bowel alteration. The patient did not have chest-pain, respiratory

Address of Correspondence: Dr. Md. Abdul Mazid, Associate Professor of Surgery, TMSS Medical College \& Rafatullah Community Hospital, Bogra. Mobile: 01914 123715. Emailamazidmilon@gmail.com

Received: 26 January, 2014

Accepted: 26 January, 2015
Acute gastric outlet obstruction is relatively uncommon and mostly due to foreign bodies related to food impaction, with meat being the most frequent culprit. The diagnostic approach to acute gastric outlet obstruction is similar to other cause of GOO. However, therapeutic options differ for each patient. The diagnosis should be made in prompt time to prevent life threatening complications due obstruction and/or effect of medication forming bezoar.

Keywords: Medication bezoar, gastric outlet obstruction, endoscopy.

(J Banagladesh Coll Phys Surg 2015; 33: 177-180)

difficulties or sweating. She had no contributing factors such as gastric surgery, psychiatric illness, diabetes mellitus but had a history of open cholecystectomy and choledicholithotomy. The patient had a history of mild dyspeptic upper abdominal pain for which she had taken anti-ulcer drugs on occasions. She took omeprazole capsule and aluminum hydroxide tablet as medication for her complaints before admission. No oral medication was given after admission to our hospital till diagnosis. On physical examination, she appeared ill, mildly anaemic, non icteric, dehydrated with tachycardia with low blood pressure. Her abdomen was soft but mildly tender over the epigastric area. No organomegally or mass was detected on examination. The rest of her examination was unremarkable. Laboratory test results did not show any notable abnormality except mild hyponatraemia.

Abdominal X-ray film was also unremarkable. Abdominal ultrasonography showed strong post acoustic shadow noted within 1st part of duodenum (Fig.1) possibly foreign body. After sufficient fluid resuscitation and gastric lavage, an upper gastrointestinal endoscopy (UGE) was performed to evaluate the cause of gastric outlet obstruction. A greenish solid impacted foreign body of tablet was detected in the first portion of the duodenum with complete obstruction (Fig. 2). The solid bezoar is extracted with biopsy forceps and dormia basket, after removal it was revealed that the bezoar was formed 
tablets of aluminum hydroxide (Fig. 3). After removal of the bezoar, the patient started to take foods and drinks as before without any pain or vomiting.

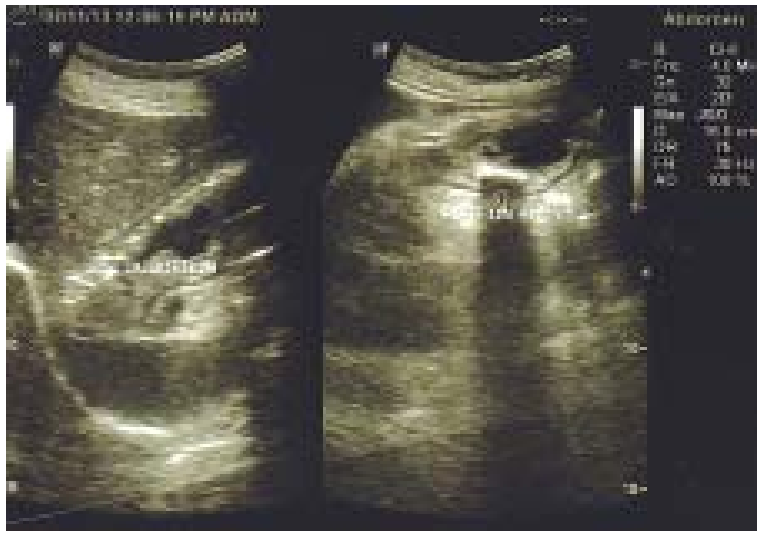

Fig.-1: USG showed foreign body within pyloric canal.

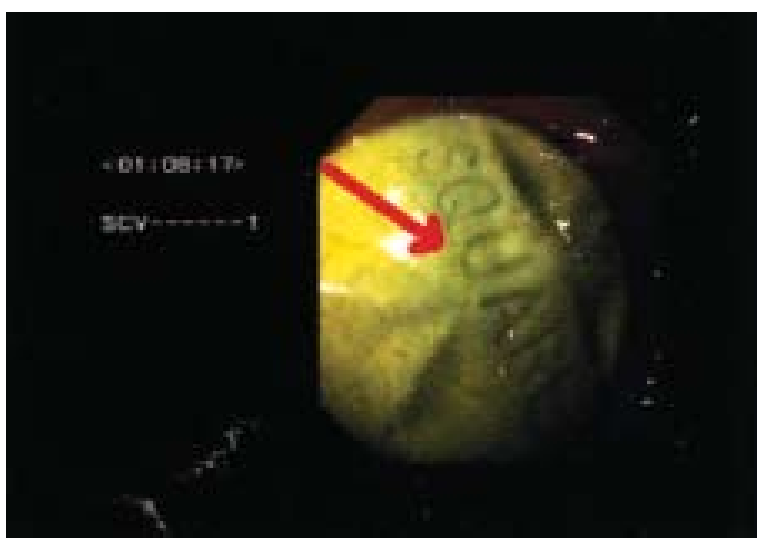

(a)

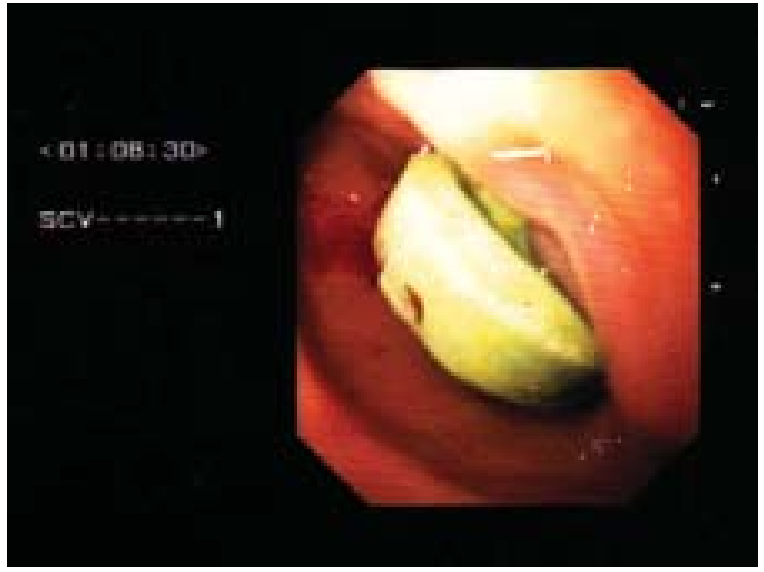

(b)

Fig.-2: (a) Endoscopic view of the bezoar, (b) Endoscopic view of the bezoar.

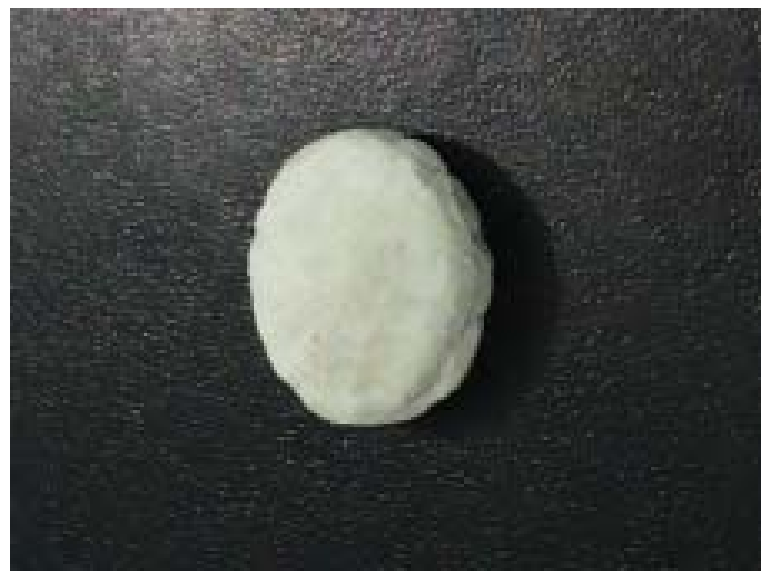

Fig.-3: Tablet after removal by endoscope.

\section{Discussion:}

Bezoars are foreign bodies in the gastrointestinal tract that increase in size by the accretion of nonabsorbable food or fibers. Interestingly, the term "bezoar" is derived from Arabic badzehr or from Persian pâd-zahr, both meaning counterpoison or antidote ${ }^{3,4}$. Bezoars are commonly found in domestic animals, and for centuries were used as charms or emollients to treat maladies as diverse as vertigo, epilepsy, and leprosy ${ }^{5}$. Although the prevalence of bezoars in humans is low, if treatment is not administered, associated mortality rates may be as high as $30 \%$ primarily because of gastrointestinal bleeding, obstruction, or perforation ${ }^{3}$. The first description of a postmortem human bezoar was by Swain in $1854^{6}$. The pathologic report of bezoars in humans was largely unrecognized, however, until 1939, when DeBakey and Ochsner reported 311 cases $^{7}$.

Gastric bezoars are usually formed by indigestible plant material (phytobezoars), ingested hair (trichobezoars), conglomerations of medications or medication vehicles (pharmacobezoars), or the combination of any of the above $^{8}$.

Medication bezoars occur within the digestive tract and are composed of medications and/or medication vehicles. Rarely, however, is bezoar formation solely due to a medication. In nearly all reported cases the patient had one or more significant risk factors that contributed to bezoar formation. They most often occur in patients with a previous history of a gastric operation and are detected in up to $20 \%$ of patients who have undergone gastric antrectomy. Reduction of gastric acidity, poor gastric mixing, psychiatric illness, coeliac 
disease and metabolic disorders such as uraemia have also been implicated as contributory factors ${ }^{5,9}$. Medications reported to cause bezoars include aluminum hydroxide gel, enteric-coated aspirin, sucralfate, guar gum, cholestyramine, enteral feeding formulas, psyllium preparations, nifedipine XL, and meprobamate ${ }^{1}$. Bowel hypoactivity, dehydration, and concomitant use of anticholinergics and narcotis appear to contribute to the propensity for bezoar formation by aluminum hydroxide gel and Isocal ${ }^{1}$. The hygroscopic properties of psyllium and guar gum appear to contribute to their propensity to form bezoars. Insolubility of the carrying vehicle of enteric-coated aspirin and nifedipine is the setting in which these medications form bezoars ${ }^{1}$. In our case, tablet of aluminum hydroxide to cause the bezoar. The exact method by which medications bezoars form is dependent on the particular type or combination of medications involved. Bezoar formation may be associated with significant complications for the patient due to the presence of the bezoar and because of the effects of the medication within the bezoar.

Gastric outlet obstruction (GOO) is a well known condition in Gastrointestinal practice. But acute gastric outlet obstruction is relatively uncommon. In general, most upper gastrointestinal tract obstructions are due to foreign bodies related to food impaction, with meat being the most frequent culprit ${ }^{10}$. Anybody presented with features of acute gastric outlet obstruction, a meticulous history of ingestion including drugs and others are very important especially in children, mentally retarded and old patient and in those who had previous abdominal operations. The diagnostic approach is similar to the other causes of the GOO. Upper gastrointestinal endoscopy, is not only an investigating tool but also a good option for therapeutic approach for acute gastric outlet obstruction. Ultrasonography and computerized tomography may be done. Treatment of medication bezoars depends largely on the location, cause and complications of the bezoar. To date, treatment modalities includes endoscopic removal involves fragmenting the larger bezoar by water flushes, direct suction, large polypectomy snare, biopsy forceps, electrosurgical knife, mechanical or extracorporeal lithotripsy and Nd:YAG laser ${ }^{11}$. Small bezoar may be amenable to nasogastric lavage or suction, a clear liquid diet, and the use of prokinetic agents ${ }^{12}$. Dissolution trials with cola and acetylcysteine may also be used ${ }^{11}$. A recently described technique from China incorporates a laser mini-explosive technique through an endoscope; in 100 patients, the cure rate was $100 \%{ }^{13}$. Operative intervention may be needed if endoscopic therapy fails or if there is a complication related to the bezoar such as perforation or bleeding. However, GOO due to bezoars requires early surgery as it rarely improves with conservative therapy. If surgical intervention is required, bezoar removal is commonly done by gastrotomy. For this, either a laparotomy or laparoscopy can be used. Different reports have been published for laparoscopic removal. This technique is usually preferred because of its less postoperative pain and better cosmetic results. However, the longer operating time, higher costs and retrieval problems are the disadvantages of laparoscopy ${ }^{14}$. For the presented case, endoscopic removal were done and found bezoar of tablet of aluminum hydroxide.

\section{Conclusion:}

High index of suspicion should be there to identify the medication bezoar as a cause of acute gastric outlet obstruction. The diagnosis should be made in prompt time to prevent life threatening complications due obstruction and/or effect of medication forming bezoar. Surgical intervention is required if conservative treatment or endoscopy fails or complication such as perforation or bleeding.

\section{Reference:}

1. Stack PE, Thomas E. Pharmacobezoar: an evolving new entity. Dig Dis 1995;13(6):356-364.

2. Taylor JR, Streetman DS, Castle SS. Medication bezoars: a literature review and report of a case. Ann Pharmacother 1998 Sep;32(9):940-946.

3. Williams RS. The fascinating history of bezoars. Med J Aust 1986;145:613-614.

4. http://en.wikipedia.org/wiki/Bezoar.

5. Phillips MR, Zaheer S, Drugas GT. Gastric Trichobezoar: Case Report and Literature Review. Mayo Clin Proc 1998;73:653-656.

6. Chishoim EM, LeongHT, Chung SC. Li AK. Phytobezoar: an uncommon cause of small bowel obstruction. Ann R Coll Surg Engl 1992;74:342-344.

7. DeBakey M, Ochsner A. Bezoars and concretions: a comprehensive review of the literature with analysis of 
303 collected cases and a presentation of 8 additional cases. Surgery 1939;5:132-160.

8. Sanders MK. Bezoars: from mystical charms to medical and nutritional management. Pract Gastroenterol 2004;28:37-50.

9. Rowshon AHM, Uddin AKMR. Capsule-strip- An unusual Cause of Acute Gastric Outlet Obstruction: A Case-Report. J Shaheed Suhrawardy Med Coll, 2013;5(1):67-68.

10. Conway WC, Sugawa C, Ono H, Lucas CE. Upper GI foreign body: an adult urban emergency hospital experience. Surg Endosc 2007;21:455-460.
11. Guner A, Kahraman I, Aktas A, Kece C, Reis E. Gastric outlet obstruction due to duodenal bezoar: A case report. Int J Surg C R 2012;3:523-525.

'12. Abbas TO. An Unusual Cause of Gastrointestinal Obstruction: Bezoar. Oman Med J. 2011 March; 26(2): 127-128.

13. Huang YC, Liu QS, Guo ZH. The use of laser ignited miniexplosive technique in treating 100 cases of gastric bezoars. Zhonghua Nei Ke Za Zhi. 1994;33(3):172-174.

14. Sharma D, Srivastava M, Babu R, Anand R, Rohtagi A, Thomas S. Laparoscopic treatment of gastric bezoar. J Soci Laparoendo Surg 2010;14:263-267. 